\title{
Improved efficacy and safety of low-dose oxaliplatin/pegylated liposomal doxorubicin/S-1 regimen in advanced gastric cancer: a cohort study
}

\author{
Jian Ma, Min Xiao, Xiaoqian Li, Qiu Zhao, Wei Ye, Wenjing Ji, Yang Ling, Quanliang Yang \\ Department of Oncology, Changzhou Tumor Hospital Affiliated to Soochow University, Changzhou, China \\ Contributions: (I) Conception and design: J Ma, Q Yang, Y Ling; (II) Administrative support: Y Ling, Q Yang; (III) Provision of study materials or \\ patients: M Xiao, X Li, Q Zhao, W Ye, W Ji; (IV) Collection and assembly of data: J Ma, Q Yang; (V) Data analysis and interpretation: J Ma; (VI) \\ Manuscript writing: All authors; (VII) Final approval of manuscript: All authors. \\ Correspondence to: Quanliang Yang. Department of Oncology, Changzhou Tumor Hospital Affiliated to Soochow University, 68 Honghe Road, Xinbei \\ District, Changzhou 213002, China. Email: azskyhorse@outlook.com.
}

\begin{abstract}
Background Dose-limiting neurotoxicity is the major side effect caused by oxaliplatin treatment. Optimization of oxaliplatin-containing chemotherapeutic regimens may potentially benefit advanced gastric cancer (AGC) patients. We tried to reduce the dose of oxaliplatin and add a drug to compensate for the efficacy. This cohort study evaluated the efficacy and side effects of low-dose oxaliplatin combined with pegylated liposomal doxorubicin and S-1 (D-SOX) as first-line treatment for AGC.

Methods: 64 AGC patients treated in our hospital between January 2015 and December 2018 were included in this study. Among them, 29 cases received standard S-1 and oxaliplatin (SOX) regimen, and 35 cases received D-SOX. Progression-free survival (PFS), overall survival (OS), response rate (RR), and safety were analyzed.

Discussion: The median PFS was 7.0 months [95\% confidence interval (CI): 5.77 to 8.23 months] in the SOX group and 9.3 months (95\% CI: 8.145 to 10.45 months) in the D-SOX group ( $\mathrm{P}=0.021)$. The median OS was 12.5 months (95\% CI: 7.00 to 17.97 months) in the SOX group and 18.7 months (95\% CI: 14.485 to 22.9 months) in the D-SOX group ( $\mathrm{P}=0.027)$. The incidence of treatment-related grade III-IV adverse events (AEs) was less than $10 \%$. The RRs to these 2 regimens were similar $(\mathrm{P}=0.609)$. The incidence of neurotoxicity was significantly reduced in the D-SOX group (62.8\% vs. $82.7 \%)$.
\end{abstract}

Conclusions: Low-dose oxaliplatin combined with S-1 and pegylated liposomal doxorubicin (PLD) regimen improved OS and PFS, while exhibiting better toxicity profile as compared with standard SOX regimen for AGC.

Keywords: Oxaliplatin; pegylated liposomal doxorubicin (PLD); advanced gastric cancer (AGC); SOX

Submitted Nov 02, 2021. Accepted for publication Dec 22, 2021.

doi: 10.21037/apm-21-3584

View this article at: https://dx.doi.org/10.21037/apm-21-3584

\section{Introduction}

In 2018, gastric cancer (GC) was the fifth most commonly diagnosed cancer type and the third leading cause of cancerrelated death, comprising $8.2 \%$ of all cancer-related deaths (1).

Chemotherapy remains the mainstream option for patients who have metastases at the time of diagnosis and are past the window of opportunity for surgery. Fluorouracil (5-FU; capecitabine, S-1, etc.), paclitaxel, and platinum (cisplatin, oxaliplatin, etc.) are the most commonly used chemotherapeutic drugs. The National Comprehensive Cancer Network (NCCN) guidelines recommend 2-drug regimens (fluorouracil + platinum). In Asia and North America, S-1 and capecitabine are the mostly used drugs, respectively, and have been shown to have equivalent efficacy (2). On account of significant side effects, a 3-drug regimen is only reserved for patients who are in good 
condition and can generally tolerate the toxic reaction $(3,4)$. According to the V325 clinical study (3), adding docetaxel to cisplatin and 5-FU (CF) significantly improved timeto-progression (TTP), survival, and response rate (RR) in $\mathrm{GC}$, but resulted in some increase in toxicity. However, in a recent clinical trial, JCOG1013 (4), the addition of docetaxel to cisplatin and S-1 did not improve overall survival (OS) in chemotherapy-naïve Japanese patients with advanced gastric cancer (AGC). Anthracycline, once the first choice for perioperative chemotherapy for GC (5), is also used in chemotherapy for AGC (6). However, due to the unique side effects of anthracycline and the development of taxane, the application of anthracycline is greatly restricted. A network meta-analysis (7) showed anthracycline-containing triplets and taxane-containing CF (TCF) triplet regimen showed no benefit over 2-drug regimens containing 5-FU in OS and progression-free survival (PFS). At present, the recommended level of anthracycline in NCCN chemotherapy for AGC is only $2 \mathrm{~B}$.

Oxaliplatin is superior to cisplatin in that it is at least as effective as cisplatin but has preferable toxicity and tolerability profiles (8). However, increased neurotoxicity remains challenging for patients receiving the treatment, and no specific preventive measure is currently available. Decrease in cumulative dose may reduce the occurrence of neurotoxicity, but this approach may sacrifice the efficacy of oxaliplatin (9). Although cumulative dose is clearly critical in determining the development of oxaliplatin-induced neurotoxicity, the possibility that chronic effects may be minimized through the use of a low single-infusion dosage requires further investigation. Therefore, optimization of an oxaliplatin-containing regimen could potentially improve therapeutic outcomes by retaining the efficacy while limiting neurotoxicity.

The unique delivering mechanism of pegylated liposomal doxorubicin (PLD) provides benefit by reducing the side effects and enhancing targeting at the disease site (10-12), but its application in GC is rarely reported. Over the past 2 decades, there have only been 2 phase II clinical trials, both have involved a small AGC sample size $(13,14)$ and the most recent trial was conducted nearly 10 years ago.

In this retrospective study, we intended to re-evaluate the effectiveness of PLD in combination with S-1 that are currently commonly used by adding an appropriate dose of PLD to compensate for the possible reduction in efficacy caused by decrease in oxaliplatin dose. We analyzed and compared the data of the patients who received PLD combined with S-1 and low-dose oxaliplatin (D-SOX) with those who received the standard SOX regimen for the same time period.

We present the following article in accordance with the STROBE reporting checklist (available at https://dx.doi. org/10.21037/apm-21-3584).

\section{Methods}

\section{Patients}

We recruited AGC patients who received SOX or PLD combined with S-1 and low-dose oxaliplatin (D-SOX) as first-line chemotherapy in our hospital from 2015 to 2018 to the study.

The selection criteria were as follows: (I) $\geq 20$ years old; (II) Eastern Cooperative Oncology Group (ECOG) performance status $\leq 2$; (III) pathologically confirmed locally advanced unresectable or metastatic GC; (IV) human epidermal growth factor receptor 2 (HER-2) negative: immunohistochemistry HER-2 (-) or (+); (++) and FISH (-); (V) no chemotherapy and radiotherapy after diagnosis (adjuvant chemotherapy was allowed if performed more than 6 months ago); (VI) the expected survival time of the patient was $\geq 3$ months; (VII) patients with measurable disease; (VIII) liver and bone marrow function satisfying absolute neutrophil count $\geq 1,000 / \mathrm{mm}^{3}$, platelet $\geq 7.5 \times 10^{4} / \mathrm{mm}^{3}$, total bilirubin $\leq 1.5 \mathrm{mg} / \mathrm{dL}$, aspartate transaminase (AST) $\leq 100 \mathrm{IU} / \mathrm{L}$, alanine transaminase (ALT) $\leq 100 \mathrm{IU} / \mathrm{L}$ (total bilirubin $\leq 2.0 \mathrm{mg} / \mathrm{dL}$, AST $\leq 200 \mathrm{IU} / \mathrm{L}, \mathrm{ALT} \leq 200 \mathrm{IU} / \mathrm{L}$ in patients with liver metastasis).

All procedures performed in this study involving human participants were in accordance with the Declaration of Helsinki (as revised in 2013), and was approved by the Ethical Committee of Changzhou Tumor Hospital Affiliated to Soochow University (2017-SY-012). Written informed consent was obtained from the patients for publication of this study and any accompanying images.

\section{Treatment}

According to the inclusion criteria, a total of 64 patients were selected, including 29 patients in the SOX group and 35 patients in the D-SOX group.

The patients in the SOX regimen group received oxaliplatin $130 \mathrm{mg} / \mathrm{m}^{2} \mathrm{~d} 1, \mathrm{~S}-140 \mathrm{mg} / \mathrm{m}^{2}$ b.i.d d1-14; D-SOX regimen: PLD $25 \mathrm{mg} / \mathrm{m}^{2} \mathrm{~d} 1$, oxaliplatin $85 \mathrm{mg} / \mathrm{m}^{2}$ d1, S-1 $40 \mathrm{mg} / \mathrm{m}^{2}$ b.i.d d1-14, and both regimens had a 
21-day cycle. The treatment continued until the disease progressed or unacceptable side effects occurred, or the patient gave up the treatment. In the pre-experiment, the D-SOX regimen was well tolerated. The dose of SOX regimen referred to the NCCN guidelines.

Which scheme was used by the patient was decided by consultation between the doctor and the patient, and the factors of consideration included the patient's physical condition, economic status, treatment purpose, and the requirements for efficacy and side effects, which were not randomly assigned. This was deemed a better reflection of the medical situation in the real world.

\section{Assessments}

This retrospective analysis was conducted to compare the efficacy and safety of D-SOX regimens and SOX regimens, including objective RR (ORR), PFS, and OS. The RR was evaluated with enhanced computed tomography (CT) every 2 cycles, including chest and abdomen CT, according to the response evaluation criteria in solid tumors (RECIST) 1.1.

Complete response (CR) was defined as a disappearance of all target lesions. Any pathological lymph nodes (whether target or non-target) must have had a reduction in short axis to $<10 \mathrm{~mm}$.

Partial response (PR) was defined as an at least 30\% decrease in the sum of diameters of target lesions, taking as reference the baseline sum diameters.

Progressive disease (PD) was defined as a least $20 \%$ increase in the sum of diameters of target lesions, taking as reference the smallest sum on study (this included the baseline sum if that was the smallest on study). In addition to the relative increase of $20 \%$, the sum must also have demonstrated an absolute increase of at least $5 \mathrm{~mm}$ (note: the appearance of 1 or more new lesions was also considered progression).

Stable disease (SD) was defined as neither sufficient shrinkage to qualify for PR nor sufficient increase to qualify for $\mathrm{PD}$, taking as reference the smallest sum-diameters during the study period.

The safety was analyzed based on the National Cancer Institute Common Terminology Criteria for Adverse Events (CTCAE) version 5.0.

\section{Statistical analysis}

The $t$-test and chi-square tests were used to compare the baseline parameters of the patients $(\mathrm{P}<0.05$ was considered statistically significant). The OS was defined as the time from initiation of chemotherapy to the date of death from any cause or censored at the latest follow-up for surviving patients. The PFS was defined as the time from initiation of chemotherapy to the date of disease progression or death, and survivors without disease progression were censored at the last contact. The OS and PFS curves were estimated using the Kaplan-Meier method. The hazard ratio (HR) was estimated using the Cox proportional hazards model. Univariate and multivariable analyses were also performed using the Cox proportional hazards model. Univariate analysis was performed to explore prognostic factors. Statistical analyses were conducted using the software SPSS 26.0 (IBM Corp., Armonk, NY, USA).

\section{Results}

\section{Patients}

According to the inclusion criteria, a total of 64 patients were selected, including 29 patients in the SOX group and 35 patients in the D-SOX group. The participant characteristics of both groups are shown in Table 1. The baseline characteristics were generally well balanced between the 2 groups. The longest follow-up period was 2 years.

\section{Efficacy and safety}

Of the 29 patients in the SOX group, 1 (3.4\%) had CR, $8(27.6 \%)$ had PR, $19(65.5 \%)$ had SD, and $1(3.4 \%)$ progressed. Of the 35 patients in the D-SOX group, 2 (5.7\%) had CR, 11 (31.4\%) had PR, 21 (60.0\%) had $\mathrm{SD}$, and 1 (2.8\%) progressed. There was no significant difference between these 2 groups in ORR $(\mathrm{P}=0.609)$ (shown in Table 2).

The median PFS was 7.0 months [95\% confidence interval (CI): 5.77 to 8.231 months] in the SOX group and 9.3 months (95\% CI: 8.145 to 10.45 months) in the D-SOX group $(\mathrm{P}=0.021)$ (Figure 1). The median OS was 12.5 months (95\% CI: 7.00 to 17.997 months) in the SOX group and 18.7 months (95\% CI: 14.48 to 22.91 months) in the D-SOX group $(\mathrm{P}=0.027)$ (Figure 2). Univariate analysis showed an association between OS and therapy regimens (D-SOX vs. SOX, $\mathrm{P}=0.030$ ). Multivariate analysis of variance suggested that $\mathrm{OS}$ was related to chemotherapy regimens (D-SOX vs. SOX, HR =0.526, P=0.027, 95\% CI: 0.297 to 0.930 ), retroperitoneal lymph node metastasis, and 
Table 1 Patient characteristics

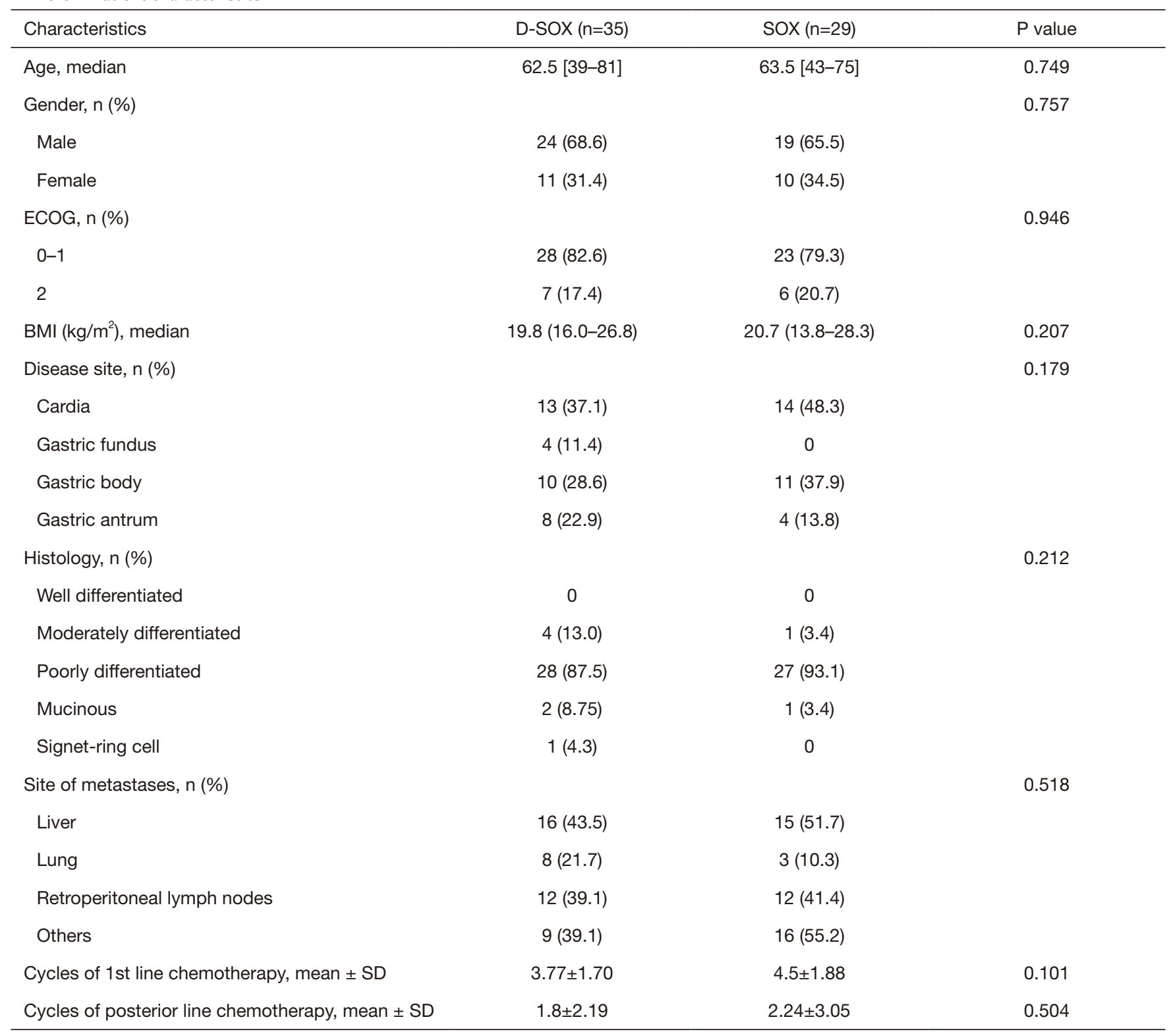

ECOG, Eastern Cooperative Oncology Group; BMI, body mass index.

Table 2 Efficacy

\begin{tabular}{lccc}
\hline Variable & D-SOX $(\mathrm{n}=35), \mathrm{n}(\%)$ & SOX $(\mathrm{n}=29), \mathrm{n}(\%)$ & $\mathrm{P}$ value \\
\hline CR & $2(5.7)$ & $1(3.4)$ & 0.571 \\
PR & $11(31.4 \%)$ & $8(27.6)$ & 0.738 \\
SD & $21(60.0)$ & $19(65.5)$ & 0.650 \\
PD & $1(2.8)$ & $1(3.4)$ & 0.705 \\
ORR & $13(37.1)$ & $9(31.0)$ & 0.609 \\
\hline
\end{tabular}

CR, complete remission; PR, partial remission; SD, stable disease; PD, progressive disease; ORR, objective response rate. 


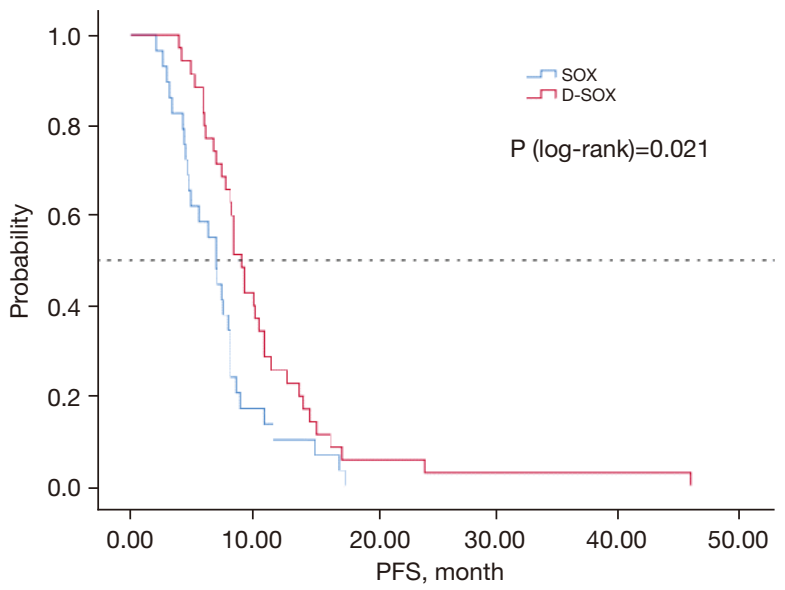

Figure 1 Kaplan-Meier progression-free survival for D-SOX versus SOX. D-SOX, pegylated liposomal doxorubicin combined with S-1 and low-dose oxaliplatin; SOX, S-1 and oxaliplatin; PFS, progression-free survival.

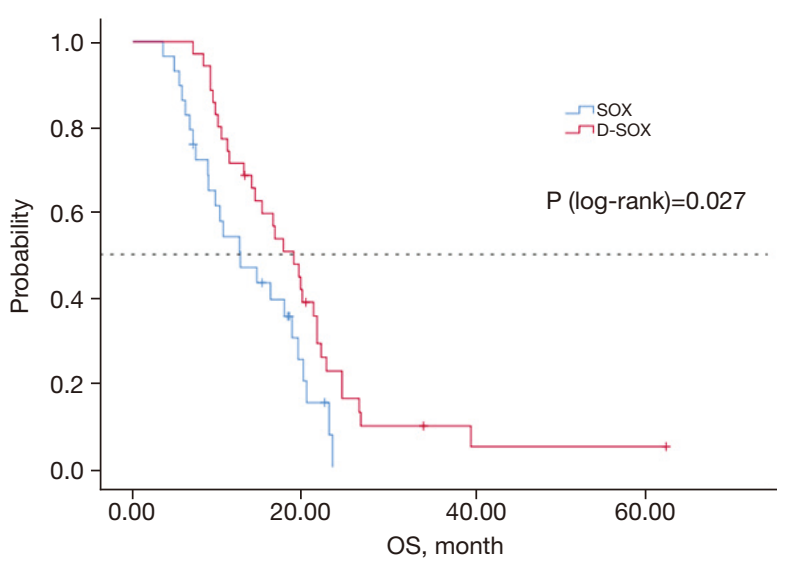

Figure 2 Kaplan-Meier overall survival for D-SOX versus SOX. D-SOX, pegylated liposomal doxorubicin combined with S-1 and low-dose oxaliplatin; SOX, S-1 and oxaliplatin; OS, overall survival.

\section{ECOG score (shown in Table 3).}

The adverse events (AEs) of all participants are summarized in Table 4. Both therapies were tolerated well by most participants, with most side effects classified as Grade 1-2 (Table 4). Among them, gastrointestinal reaction and neutropenia were common. Among the participants treated with D-SOX, the addition of PLD did not significantly increase the incidence of side effects. It is worth noting that the incidence of neurotoxicity in the D-SOX group was significantly reduced ( $60 \% v s .79 .3 \%)$, highly likely due to the use of lower doses of oxaliplatin. Secondly, the incidence of thrombocytopenia in SOX was slightly higher, which may have been related to the higher dose of oxaliplatin. No obvious cardiotoxicity was observed in both groups.

\section{Discussion}

In clinical practice, chemotherapy does not exceed the second line in most patients. Although many drugs, including irinotecan, paclitaxel, docetaxel, anthracycline, and so on, are available as second line options, only 1 of them is generally used for treatment, while a variety of potentially effective chemotherapy drugs for GC will not be ultimately used. On the other hand, the efficacy of chemotherapy for GC has not been significantly improved for many years. Therefore, it remains unclear whether reducing the dose of 1 drug and increasing the types of drugs with different mechanisms in front-second-line chemotherapy can increase the efficacy and concurrently improve patient's quality of life, which is a critical therapeutic goal in modern healthcare.

This retrospective study was designed to evaluate a modified chemotherapy regimen containing oxaliplatin, which is commonly used and associated with high incidence of neurotoxicity, and PLD. In the modified D-SOX regimen, we reduced the dose of oxaliplatin from 130 to $85 \mathrm{mg} / \mathrm{m}^{2}$, plus PLD, $25 \mathrm{mg} / \mathrm{m}^{2}$ every 21 days, and S-1 (40 $\mathrm{mg} / \mathrm{m}^{2}$ b.i.d $\left.\mathrm{d} 1-14\right)$, and compared its efficacy and toxicity profile with the traditional SOX regimen. The median PFS was 7.0 months (95\% CI: 5.77 to 8.231 months) in the SOX group and 9.3 months (95\% CI: 8.145 to 10.45 months) in the D-SOX group $(\mathrm{P}=0.021)$ (Figure 1). The median OS was 12.5 months (95\% CI: 7.00 to 17.997 months) in the SOX group and 18.7 months (95\% CI: 14.48 to 22.91 months) in the $\mathrm{D}-\mathrm{SOX}$ group $(\mathrm{P}=0.027)$. In the $\mathrm{D}-\mathrm{SOX}$ group, both $\mathrm{PFS}$ and OS were prolonged. The side effects were not significantly increased or aggravated.

The results are encouraging, but the number of samples involved was small. Another limitation is that the selection of cases did not strictly follow the principle of randomization. In real-world clinical practice, many factors affect doctors and patients' choice, even within the scope of the guidelines. Patients with good general condition may be more inclined to be treated with 3 drugs. This may also be one of the reasons for the significant prolongation of OS in the D-SOX group, where such an extension of survival was beyond expectations. It is hoped that there will be well- 
Table 3 Cox regression

\begin{tabular}{|c|c|c|c|c|c|c|c|c|c|}
\hline \multirow{3}{*}{ Variable } & \multicolumn{3}{|c|}{ PFS } & \multicolumn{6}{|c|}{ os } \\
\hline & \multicolumn{3}{|c|}{ Univariate Cox regression model } & \multicolumn{3}{|c|}{ Univariate Cox regression model } & \multicolumn{3}{|c|}{ Multivariate Cox regression mode } \\
\hline & $\mathrm{HR}$ & $95 \% \mathrm{Cl}$ & $P$ value & $\mathrm{HR}$ & $95 \% \mathrm{Cl}$ & $P$ value & $\mathrm{HR}$ & $95 \% \mathrm{Cl}$ & $P$ value \\
\hline Gender (male vs. female) & 0.639 & $0.369-1.105$ & 0.109 & 0.937 & $0.525-1.671$ & 0.825 & & & \\
\hline Age (<65 vs. $\geq 65)$ & 0.827 & $0.499-1.373$ & 0.463 & 1.39 & $0.802-2.410$ & 0.240 & & & \\
\hline BMI $\left(\mathrm{kg} / \mathrm{m}^{2}\right)$ & 0.987 & $0.896-1.087$ & 0.788 & 0.996 & $0.901-1.110$ & 0.932 & & & \\
\hline \multicolumn{10}{|l|}{ Disease site } \\
\hline Cardia & 1.0 & & & 1.0 & & & & & \\
\hline Gastric fundus & 1.347 & $0.461-3.937$ & 0.587 & 1.925 & $0.649-5.709$ & 0.237 & & & \\
\hline \multicolumn{10}{|l|}{ Histology } \\
\hline Adenocarcinoma & 1.0 & & & 1.0 & & & & & \\
\hline Mucinous & 3.694 & $1.094-12.476$ & 0.035 & 2.879 & $0.865-9.581$ & 0.085 & & & \\
\hline Signet-ring cell & 2.482 & $0.753-8.180$ & 0.135 & 1.538 & $0.475-4.982$ & 0.473 & & & \\
\hline $\begin{array}{l}\text { Differentiation } \\
(1=\text { moderately; } 2=\text { poorly })\end{array}$ & 0.757 & $0.323-2.049$ & 0.662 & 0.554 & $0.196-1.569$ & 0.266 & & & \\
\hline \multicolumn{10}{|l|}{ Site of metastases } \\
\hline Liver & 0.913 & $0.554-1.507$ & 0.723 & 0.805 & $0.470-1.379$ & 0.43 & & & \\
\hline $\begin{array}{l}\text { Cycles of } 1 \text { st line } \\
\text { chemotherapy (>2 vs. } \leq 2)\end{array}$ & 0.738 & $0.424-1.284$ & 0.282 & 0.754 & $0.407-1.395$ & 0.368 & & & \\
\hline $\begin{array}{l}\text { Posterior line chemotherapy } \\
\text { (yes vs. no) }\end{array}$ & - & - & - & 0.840 & $0.494-1.430$ & 0.521 & & & \\
\hline
\end{tabular}

PFS, progression-free survival; OS, overall survival; HR, hazard ratio; Cl, confidence interval; ECOG, Eastern Cooperative Oncology Group; BMI, body mass index.

designed clinical trials to verify the effectiveness of the $\mathrm{D}-\mathrm{SOX}$ regimen in the future.

Our results demonstrated 3 advantages in limiting chemotherapy-induced side effects. First, lowering singleinfusion dosage and consequent cumulative dose of oxaliplatin significantly decreased the incidence of grade 1-2 neurotoxicity. Neurotoxicity is the most worrying side effect caused by repeated use of oxaliplatin. Oxaliplatin- induced neurotoxicity is unique among platinum analogues, producing a distinctive spectrum of symptoms in both acute and chronic presentations. Acute neurotoxicity develops immediately following infusion and is characterized by transient paraesthesia and muscular spasms in the limbs and perioral region (including jaw spasm), typically triggered or exacerbated by cold exposure $(15,16)$. Acute, transient symptoms occur in $95 \%$ of patients with evidence of 
Table 4 Side effects

\begin{tabular}{|c|c|c|c|c|c|}
\hline Variable & \multicolumn{2}{|c|}{ D-SOX (n=35), n (\%) } & \multicolumn{2}{|c|}{$\operatorname{sOX}(n=29), n(\%)$} & $P$ value \\
\hline Diarrhea & $3(8.6)$ & 0 & $2(6.9)$ & 0 & 0.59 \\
\hline Nausea/vomiting & $16(45.7)$ & $2(5.7)$ & $13(44.8)$ & $2(6.9)$ & 0.981 \\
\hline Mucositis & $1(2.8)$ & 0 & 0 & 0 & 0.547 \\
\hline Asthenia & $4(11.4)$ & 0 & $3(10.3)$ & 0 & 0.607 \\
\hline Live toxicity & $1(2.8)$ & 0 & $1(3.4)$ & 0 & 0.705 \\
\hline Renal toxicity & 0 & 0 & 0 & 0 & - \\
\hline Peripheral sensory neuropathy & $21(60.0)$ & $1(2.8)$ & $23(79.3)$ & $1(3.4)$ & 0.078 \\
\hline Febrile neutropenia & 0 & $2(5.7)$ & $1(3.4)$ & $2(6.9)$ & 0.41 \\
\hline Anemia & $4(11.4)$ & 0 & $4(13.8)$ & 0 & 0.534 \\
\hline Alopecia & $3(8.6)$ & 0 & 0 & 0 & 0.157 \\
\hline
\end{tabular}

peripheral nerve hyperexcitability on electromyography (17). In contrast, chronic neurotoxicity predominantly produces sensory dysfunction with distal paraesthesia progressing to sensory ataxia and functional impairment $(15,16)$. Severe neurotoxicity is strongly dose-dependent and occurs in $10-20 \%$ of patients at cumulative doses of $750 \mathrm{mg} / \mathrm{m}^{2}(18-$ 20 ) and up to $50 \%$ of patients at higher doses. Symptoms may persist long-term $(9,21,22)$ representing a significant limitation to treatment, as end-organ neurotoxicity and neuropathy may require discontinuation of effective therapy. More critically, the development of neuropathy poses a problematic issue in the setting of adjuvant therapy, where long term neurotoxicity is an unacceptable outcome. Moreover, for adjuvant chemotherapy, drugs such as oxaliplatin and cisplatin are the inevitable choice, so for patients with recurrence after adjuvant therapy, the cumulative dose of oxaliplatin will be higher. Sensory abnormalities may persist for months, or even years after the cessation of chemotherapy and recovery is usually incomplete (23). Effective treatment of established chemotherapy-induced peripheral neuropathy (CIPN), however, has yet to be determined $(23,24)$. Krishnan (9) showed that cumulative dose is clearly critical in determining the development of oxaliplatin-induced neurotoxicity, and the possibility that chronic effects may be minimized by using a lower single-infusion dosage requires further study.

Second, the D-SOX regimen contains PLD, a form superior to doxorubicin with significantly reduced toxicity and side effects, especially cardiotoxicity, likely due to the low peak concentration of free doxorubicin in plasma after administration of PLD and the trend of accumulation of liposome drugs in the myocardium (25). This improved safety profile is attributable to PLD's unique mechanism (25-28): (I) low immunogenicity, limited side effects, and amphiphilic molecules with improved solubility; (II) almost $100 \%$ of liposomes exist in the form of liposomes in the blood circulation; (III) after liposomes are biodegraded, the drug is released slowly, and the circulation time of the drug in the body is prolonged (T1/2 55-70 h); (IV) reasonable liposome size-the average particle size is about $90 \mathrm{~nm}$; (V) enhanced permeability and retention (EPR) effect, tumor vascular permeability increases, and liposomes are smaller, which can pass through defective blood vessels, thus increasing the local drug concentration in tumor tissue about 20 times that of regular doxorubicin. It was not until a significantly higher lifetime dose was reached that the cardiotoxicity of the nanoparticle preparation PLD was observed (29,30). Gill et al. (31) pointed out that PLD has no obvious cardiotoxicity, which is shown by the stability of left ventricular ejection fraction (LVEF) even after high cumulative dose treatment. In the absence of other risk 
factors, routine monitoring of LVEF does not seem to be necessary or cost-effective.

Third, the D-SOX regimen contains a relatively lower dose of PLD compared with recommended doses in other cancer types $(32,33)$, further minimizing doxorubicininduced side effects. In our study, the cumulative dose of PLD, even after 6 standard cycles of chemotherapy, was only $150 \mathrm{mg} / \mathrm{m}^{2}$. At this dose level, no serious adverse reactions were observed, including cardiotoxicity, mucositis, palmar-plantar erythrodysesthesia (PPE), and so on, and the incidence of alopecia was rare. Hypersensitivity reaction (HSR), acute hypersensitivity (infusion) reactions referred to as complement activation-related pseudoallergy (CARPA) (34-36) occurred in one patient. Reinfusing and reducing the infusion rate after pausing for $2 \mathrm{~h}$ resolved this HSR.

At present, there is no clear evidence that PLD has a better efficacy than traditional doxorubicin. Meta-analysis showed (37) that in the case of ovarian cancer, the PFS benefit of the PLD-based regimen was significant, although the OS did not improve. Another meta-analysis (38) showed that the efficacy of liposome (liposome anthracyclines, liposome cisplatin, and so on) was not different from that of conventional chemotherapy, and even the subgroup of liposome doxorubicin did not show an obvious advantage. At the same time, this article conducted a meta-analysis of 11 preclinical studies of liposome anthracyclines. In contrast to the clinical results, animal studies showed that the survival rate of mice treated with PLD was significantly higher than that of traditional anthracyclines (HR 0.39; 95\% CI: 0.27 to 0.56). It seems that the potential of PLD has not been fully exploited.

There has been wide use of PLD in hematological tumors, ovarian cancer, and breast cancer (30,39-42), but not in the treatment of GC. A phase II clinical trial in 2001 (33) suggested that liposome doxorubicin alone had no significant antineoplastic activity in GC. Recchia et al. (43) used PLD combined with oxaliplatin in the salvage treatment of GC, with a median PFS of 5.8 months and OS of 9.2 months. In 2005, the phase II clinical trial by Gnad-Vogt et al. (13) showed that PLD combined with mitomycin and 5-FU achieved a RR of $47 \%$ in the firstline treatment of GC, with a median PFS of 8.4 months and an OS 14.7 months. In 2011, Cascinu et al. (14) conducted a phase II clinical trial in which PLD, cisplatin, and 5-FU were used in the first-line treatment of GC, compared with mitomycin C, cisplatin, and 5-FU. The ORR, time-toprogression (TTP), and OS were superior to the control group. This trial was once cited in the NCCN guidelines and later deleted. In the past 9 years, there has been no further clinical study.

In the present study, we demonstrated that the modified D-SOX regimen significantly improved PFS and OS in AGC patients as compared with the SOX regimen. The root cause highly likely lies in the chemotherapy regimen.

With the development of molecular targeted therapy and immunotherapy, there has been a significant decrease in the number of patients receiving chemotherapy exceeding the second line in clinical practice. Therefore, initiating as many effective drugs as possible in first-line and second-line therapy may benefit patients to the greatest extent. Taking into account the limitation of side effects, combination of 3 drugs at low doses may represent a promising option, as our study has demonstrated.

In conclusion, PLD combined with $\mathrm{S}-1$ and low-dose oxaliplatin might be a better tolerated and more effective treatment for AGC.

\section{Acknowledgments}

We thank the staff of the Department of Oncology of Changzhou Tumor Hospital Affiliated to Soochow University.

Funding: This study was supported by Changzhou Science \& Technology Program, China (CJ20160049).

\section{Footnote}

Reporting Checklist: The authors have completed the STROBE reporting checklist. Available at https://dx.doi. org/10.21037/apm-21-3584

Data Sharing Statement: Available at https://dx.doi. org/10.21037/apm-21-3584

Conflicts of Interest: All authors have completed the ICMJE uniform disclosure form (available at https://dx.doi. org/10.21037/apm-21-3584). The authors have no conflicts of interest to declare.

Ethical Statement: The authors are accountable for all aspects of the work in ensuring that questions related to the accuracy or integrity of any part of the work are appropriately investigated and resolved. All procedures performed in this study involving human participants were in accordance with the Declaration of Helsinki (as revised in 2013) and was approved by the Ethical Committee 
of Changzhou Tumor Hospital Affiliated to Soochow University (2017-SY-012). Written informed consent was obtained from the patients for publication of this study and any accompanying images.

Open Access Statement: This is an Open Access article distributed in accordance with the Creative Commons Attribution-NonCommercial-NoDerivs 4.0 International License (CC BY-NC-ND 4.0), which permits the noncommercial replication and distribution of the article with the strict proviso that no changes or edits are made and the original work is properly cited (including links to both the formal publication through the relevant DOI and the license). See: https://creativecommons.org/licenses/by-nc-nd/4.0/.

\section{References}

1. Bray F, Ferlay J, Soerjomataram I, et al. Global cancer statistics 2018: GLOBOCAN estimates of incidence and mortality worldwide for 36 cancers in 185 countries. CA Cancer J Clin 2018;68:394-424.

2. Wu R, Shen Z, Yu N, et al. Efficacy and safety of liposome-paclitaxel/liposome-paclitaxel combined with S-1 in 17 advanced gastric cancer pa-tients with poor performance status. Transl Cancer Res 2019;8:1690-8.

3. Van Cutsem E, Moiseyenko VM, Tjulandin S, et al. Phase III study of docetaxel and cisplatin plus fluorouracil compared with cisplatin and fluorouracil as first-line therapy for advanced gastric cancer: a report of the V325 Study Group. J Clin Oncol 2006;24:4991-7.

4. Yamada Y, Boku N, Mizusawa J, et al. Docetaxel plus cisplatin and S-1 versus cisplatin and S-1 in patients with advanced gastric cancer (JCOG1013): an open-label, phase 3, randomised controlled trial. Lancet Gastroenterol Hepatol 2019;4:501-10.

5. Cunningham D, Allum WH, Stenning SP, et al. Perioperative chemotherapy versus surgery alone for resectable gastroesophageal cancer. N Engl J Med 2006;355:11-20.

6. Cunningham D, Starling N, Rao S, et al. Capecitabine and oxaliplatin for advanced esophagogastric cancer. N Engl J Med 2008;358:36-46.

7. Ter Veer E, Haj Mohammad N, van Valkenhoef G, et al. The Efficacy and Safety of First-line Chemotherapy in Advanced Esophagogastric Cancer: A Network Metaanalysis. J Natl Cancer Inst 2016. doi: 10.1093/jnci/ djw166.

8. Montagnani F, Turrisi G, Marinozzi C, et al. Effectiveness and safety of oxaliplatin compared to cisplatin for advanced, unresectable gastric cancer: a systematic review and meta-analysis. Gastric Cancer 2011;14:50-5.

9. Krishnan AV, Goldstein D, Friedlander M, et al. Oxaliplatin-induced neurotoxicity and the development of neuropathy. Muscle Nerve 2005;32:51-60.

10. Gardner ER, Dahut WL, Scripture CD, et al. Randomized crossover pharmacokinetic study of solvent-based paclitaxel and nab-paclitaxel. Clin Cancer Res 2008;14:4200-5.

11. Symon Z, Peyser A, Tzemach D, et al. Selective delivery of doxorubicin to patients with breast carcinoma metastases by stealth liposomes. Cancer 1999;86:72-8.

12. Gabizon A, Shmeeda H, Grenader T. Pharmacological basis of pegylated liposomal doxorubicin: impact on cancer therapy. Eur J Pharm Sci 2012;45:388-98.

13. Gnad-Vogt SU, Hofheinz RD, Saussele S, et al. Pegylated liposomal doxorubicin and mitomycin $\mathrm{C}$ in combination with infusional 5 -fluorouracil and sodium folinic acid in the treatment of advanced gastric cancer: results of a phase II trial. Anticancer Drugs 2005;16:435-40.

14. Cascinu S, Galizia E, Labianca R, et al. Pegylated liposomal doxorubicin, 5-fluorouracil and cisplatin versus mitomycin-C, 5-fluorouracil and cisplatin for advanced gastric cancer: a randomized phase II trial. Cancer Chemother Pharmacol 2011;68:37-43.

15. Gamelin E, Gamelin L, Bossi L, et al. Clinical aspects and molecular basis of oxaliplatin neurotoxicity: current management and development of preventive measures. Semin Oncol 2002;29:21-33.

16. Grothey A. Oxaliplatin-safety profile: neurotoxicity. Semin Oncol 2003;30:5-13.

17. Wilson RH, Lehky T, Thomas RR, et al. Acute oxaliplatininduced peripheral nerve hyperexcitability. J Clin Oncol 2002;20:1767-74.

18. André T, Bensmaine MA, Louvet C, et al. Multicenter phase II study of bimonthly high-dose leucovorin, fluorouracil infusion, and oxaliplatin for metastatic colorectal cancer resistant to the same leucovorin and fluorouracil regimen. J Clin Oncol 1999;17:3560-8.

19. Armand JP, Boige V, Raymond E, et al. Oxaliplatin in colorectal cancer: an overview. Semin Oncol 2000;27:96-104.

20. de Gramont A, Figer A, Seymour M, et al. Leucovorin and Fluorouracil With or Without Oxaliplatin as First-Line Treatment in Advanced Colorectal Cancer. J Clin Oncol 2000;18:2938-47.

21. Pietrangeli A, Leandri M, Terzoli E, et al. Persistence of high-dose oxaliplatin-induced neuropathy at long-term 
follow-up. Eur Neurol 2006;56:13-6.

22. Land SR, Kopec JA, Cecchini RS, et al. Neurotoxicity from oxaliplatin combined with weekly bolus fluorouracil and leucovorin as surgical adjuvant chemotherapy for stage II and III colon cancer: NSABP C-07. J Clin Oncol 2007;25:2205-11.

23. Ibrahim EY, Ehrlich BE. Prevention of chemotherapyinduced peripheral neuropathy: A review of recent findings. Crit Rev Oncol Hematol 2020;145:102831.

24. Wolf S, Barton D, Kottschade L, et al. Chemotherapyinduced peripheral neuropathy: prevention and treatment strategies. Eur J Cancer 2008;44:1507-15.

25. Gabizon A, Martin F. Polyethylene glycol-coated (pegylated) liposomal doxorubicin. Rationale for use in solid tumours. Drugs 1997;54 Suppl 4:15-21.

26. Sadzuka Y, Sugiyama I, Tsuruda T, et al. Characterization and cytotoxicity of mixed polyethyleneglycol modified liposomes containing doxorubicin. Int J Pharm 2006;312:83-9.

27. Caponigro F, Comella P, Budillon A, et al. Phase I study of Caelyx (doxorubicin HCL, pegylated liposomal) in recurrent or metastatic head and neck cancer. Ann Oncol 2000;11:339-42.

28. Lyseng-Williamson KA, Duggan ST, Keating GM. Pegylated liposomal doxorubicin: a guide to its use in various malignancies. BioDrugs 2013;27:533-40.

29. Uyar D, Kulp B, Peterson G, et al. Cardiac safety profile of prolonged ( $>$ or $=6$ cycles) pegylated liposomal doxorubicin administration in patients with gynecologic malignancies. Gynecol Oncol 2004;94:147-51.

30. O'Brien ME, Wigler N, Inbar M, et al. Reduced cardiotoxicity and comparable efficacy in a phase III trial of pegylated liposomal doxorubicin $\mathrm{HCl}$ (CAELYX/Doxil) versus conventional doxorubicin for first-line treatment of metastatic breast cancer. Ann Oncol 2004;15:440-9.

31. Gill SE, Savage K, Wysham WZ, et al. Continuing routine cardiac surveillance in long-term use of pegylated liposomal doxorubicin: is it necessary? Gynecol Oncol 2013;129:544-7.

32. Gordon AN, Fleagle JT, Guthrie D, et al. Recurrent epithelial ovarian carcinoma: a randomized phase III study of pegylated liposomal doxorubicin versus topotecan. J Clin Oncol 2001;19:3312-22.

33. Thomas AL, O'Byrne K, Furber L, et al. A phase II study of caelyx, liposomal doxorubicin: lack of activity in patients with advanced gastric cancer. Cancer Chemother Pharmacol 2001;48:266-8.

34. Szebeni J. Complement activation-related pseudoallergy: a stress reaction in blood triggered by nanomedicines and biologicals. Mol Immunol 2014;61:163-73.

35. Szebeni J, Storm G. Complement activation as a bioequivalence issue relevant to the development of generic liposomes and other nanoparticulate drugs. Biochem Biophys Res Commun 2015;468:490-7.

36. Szebeni J, Muggia F, Gabizon A, et al. Activation of complement by therapeutic liposomes and other lipid excipient-based therapeutic products: prediction and prevention. Adv Drug Deliv Rev 2011;63:1020-30.

37. Staropoli N, Ciliberto D, Botta C, et al. Pegylated liposomal doxorubicin in the management of ovarian cancer: a systematic review and metaanalysis of randomized trials. Cancer Biol Ther 2014;15:707-20.

38. Petersen GH, Alzghari SK, Chee W, et al. Meta-analysis of clinical and preclinical studies comparing the anticancer efficacy of liposomal versus conventional non-liposomal doxorubicin. J Control Release 2016;232:255-64.

39. Orlowski RZ, Nagler A, Sonneveld P, et al. Randomized phase III study of pegylated liposomal doxorubicin plus bortezomib compared with bortezomib alone in relapsed or refractory multiple myeloma: combination therapy improves time to progression. J Clin Oncol 2007;25:3892-901.

40. Pujade-Lauraine E, Wagner U, Aavall-Lundqvist E, et al. Pegylated liposomal Doxorubicin and Carboplatin compared with Paclitaxel and Carboplatin for patients with platinum-sensitive ovarian cancer in late relapse. J Clin Oncol 2010;28:3323-9.

41. Mutch DG, Orlando M, Goss T, et al. Randomized phase III trial of gemcitabine compared with pegylated liposomal doxorubicin in patients with platinum-resistant ovarian cancer. J Clin Oncol 2007;25:2811-8.

42. Gordon AN, Tonda M, Sun S, et al. Long-term survival advantage for women treated with pegylated liposomal doxorubicin compared with topotecan in a phase 3 randomized study of recurrent and refractory epithelial ovarian cancer. Gynecol Oncol 2004;95:1-8.

43. Recchia F, Candeloro G, Guerriero G, et al. Liposomal pegylated doxorubicin and oxaliplatin as salvage chemotherapy in patients with metastatic gastric cancer treated earlier. Anticancer Drugs 2010;21:559-64.

Cite this article as: Ma J, Xiao M, Li X, Zhao Q, Ye W, Ji W, Ling Y, Yang Q. Improved efficacy and safety of low-dose oxaliplatin/pegylated liposomal doxorubicin/S-1 regimen in advanced gastric cancer: a cohort study. Ann Palliat Med 2021;10(12):12821-12830. doi: 10.21037/apm-21-3584 